News

\title{
Food synergy: why you should eat a varied diet
}

\begin{abstract}
News
The body best acquires the protective benefits of fruits and vegetables when a variety is consumed rather than isolated nutrients (pills, supplements). Research suggests that nutrients, phytochemicals and fiber found in whole, plant-based foods work together synergistically -optimizing the health benefits for the body. For example, while vitamin $\mathrm{E}$ is an antioxidant associated with reducing risk for age related diseases such as cancer, eating foods rich in vitamin $\mathrm{E}$ in combination like eating broccoli with tomatoes provides more protection than either eaten alone and far more than a supplemental form of vitamin E. Or, take oatmeal for example. A rich form of soluble fiber, oats are helpful in lowering cholesterol. But our body reaps more benefits from eating oats than consuming supplemental soluble fiber because in addition to the soluble fiber oats contain folic acid, magnesium, phytochemicals and plant-based proteins that together are helpful in protecting against heart disease. So rather than focusing on one or two "super foods" (marketing term) or supplements aim for a balanced diet full of various fruits, vegetables and plant based foods. Eat food as close to its natural form as possible and do yourself a favor by watching the documentary King Corn.
\end{abstract}

\section{A few interesting facts}

Lycopene, a pigment found in tomatoes, watermelon and red bell peppers and polyphenols, plant compounds found in tea, cloves, peppermint, apples, berries, soy and cocoa powder have been shown to reduce our skin's sensitivity to UV rays, screening our skin slightly from the sun's rays. To help optimize protection during these summer months absolutely use sunscreen but also eat a diet rich in these protective compounds. Strawberries, blueberries and blackberries
Volume 3 Issue I - 2015

Lauren Zimmerman
Registered Dietitian, Summerville Medical Center, USA

Correspondence: Lauren Zimmerman, Registered Dietitian for cardiac and preventative health, Summerville Medical Center, 295 Midland Pkwy, Summerville, SC 29485, United States, Email laurenannzimmerman@yahoo.com

Received: May 21, 2015 | Published: September 3, 2015

rich in antioxidants help protect brain cells from damage caused by free radicals, thus helping to preserve memory and prevent cognitive decline. Antioxidant protection is a complex system working best with a variety of nutrients and antioxidants. Fruits, vegetables, whole gains and herbs are some of the best sources.

Do you know the benefits of parsley? This herb is a concentrated source of vitamin A, C and K and full of antioxidants. Reap the benefits in just a 2 tablespoon serving. Parsley pairs nicely with chicken, turkey and fish, tastes great in omelets, or works to season carrots, cauliflower, eggplant, parsnips, potatoes, spinach, or tomatoes.

\section{Acknowledgements}

None.

\section{Conflict of interest}

The author declares no conflict of interest. 